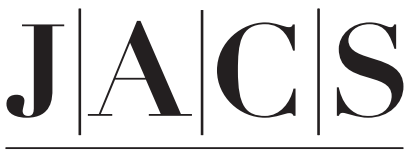

A R T I C L E S

Published on Web 04/15/2008

\title{
Sifuvirtide Screens Rigid Membrane Surfaces. Establishment of a Correlation between Efficacy and Membrane Domain Selectivity among HIV Fusion Inhibitor Peptides
}

\author{
Henri G. Franquelim, ${ }^{\dagger}$ Luís M. S. Loura, ${ }^{\ddagger, \S}$ Nuno C. Santos, ${ }^{\dagger}$ and \\ Miguel A. R. B. Castanho*,† \\ Instituto de Medicina Molecular, Faculdade de Medicina da Universidade de Lisboa, Av. Prof. \\ Egas Moniz, 1649-028 Lisboa, Portugal, Faculdade de Farmácia, Universidade de Coimbra, \\ Rua do Norte, 3000-295 Coimbra, Portugal, and Centro de Química de Évora, Rua Romão \\ Ramalho, 59, 7000-671 Évora, Portugal
}

Received December 19, 2007; E-mail: macastanho@fm.ul.pt

\begin{abstract}
Sifuvirtide, a 36 amino acid negatively charged peptide, is a novel and promising HIV fusion inhibitor, presently in clinical trials. Because of the aromatic amino acid residues of the peptide, its behavior in aqueous solution and the interaction with lipid-membrane model systems (large unilammelar vesicles) were studied by using mainly fluorescence spectroscopy techniques (both steady-state and time-resolved). No significant aggregation of the peptide was observed with aqueous solution. Various biological and nonbiological lipid-membrane compositions were analyzed, and atomic force microscopy was used to visualize phase separation in several of those mixtures. Results showed no significant interaction of the peptide, neither with zwitterionic fluid lipid membranes (liquid-disordered phase), nor with cholesterol-rich membranes (liquid-ordered phase). However, significant partitioning was observed with the positively charged lipid models $\left(K_{p}=(2.2 \pm 0.3) \times 10^{3}\right)$, serving as a positive control. Fluorescence quenching using Förster resonance acrylamide and lipophilic probes was carried out to study the location of the peptide in the membrane models. In the gel-phase DPPC (1,2-dipalmitoyl-sn-glycero-3-phosphocholine) membrane model, an adsorption of the peptide at the surface of these membranes was observed and confirmed by using Förster resonance energy-transfer experiments. These results indicate a targeting of the peptide to gelphase domains relatively to liquid-disordered or liquid-ordered phase domains. This larger affinity and selectivity toward the more rigid areas of the membranes, where most of the receptors are found, or to viral membrane, may help explain the improved clinical efficiency of sifuvirtide, by providing a local increased concentration of the peptide at the fusion site.
\end{abstract}

\section{Introduction}

Human immunodeficiency virus type 1 (HIV-1) envelope glycoprotein subunits gp120 and gp41 play a crucial role in the early steps of the viral binding and entry to the target CD4 ${ }^{+}$ host cells. ${ }^{1}$ After binding of the surface protein gp120 to CD4 and CCR5 (or CXCR4) receptors, a conformational change in the transmembrane protein gp41 is triggered, resulting in the exposure and insertion of a fusion peptide in the target cell membrane. This leads to the interaction of the heptad repeat domains of gp41 (HR1 and HR2), allowing the fusion of two membranes (viral and target cell). (See refs 2-4 and references therein).

Targeting this HR1/HR2 interaction allowed the development of HIV-1 fusion inhibitors, such as enfuvirtide (FDA-approved

\footnotetext{
Faculdade de Medicina da Universidade de Lisboa.

Universidade de Coimbra.

${ }^{\S}$ Centro de Química de Évora.

(1) Janeway, C. A. Immunobiology the immune system in health and disease, 6th ed.; Garland Science: New York, NY, 2005; p 823.

(2) Chan, D. C.; Kim, P. S. Cell 1998, 93, 681-684.

(3) Root, M. J.; Steger, H. K. Curr. Pharm. Des. 2004, 10, 1805-1825.

(4) Kilby, J. M.; Eron, J. J. N. Engl. J. Med. 2003, 348, 2228-2238.

(5) Castagna, A.; Biswas, P.; Beretta, A.; Lazzarin, A. Drugs 2005, 65 , 879-904.
}

drug) and T-1249 (development currently interrupted) ${ }^{4-7}$ (Figure 1). Both peptides present high therapeutic potency, but the natural side effects and mainly the emergence of undesired resistant strains of the virus are common in a prolonged administration of any antiretroviral drug. To overcome these problems, the discovery of more potent and less toxic inhibitors is a priority. Furthermore, the interfacial physical chemistry on the lipid surface of cells and enveloped viruses is crucial in the action of peptides that prevent such viruses from entering the cells.

Sifuvirtide (Figure 1), a novel HIV fusion inhibitor developed through structure-based drug design by FusoGen Pharmaceuticals, Inc. (Tianjin, P. R. China), ${ }^{8,9}$ overcomes several of these problems, profiling itself as a suitable and promising alternative to enfuvirtide. This 36 amino acid peptide is actually in phase II clinical trials in China and presents higher fusion inhibition

(6) Reeves, J. D.; Piefer, A. J. Drugs 2005, 65, 1747-66.

(7) Briz, V.; Poveda, E.; Soriano, V. J. Antimicrob. Chemother. 2006, 57, 619-627.

(8) Dai, S. J.; Dou, G. F.; Qiang, X. H.; Song, H. F.; Tang, Z. M.; Liu, D. S.; Liu, X. W.; Yang, L. M.; Zheng, Y. T.; Liang, Q. Acta Pharmacol. Sin. 2005, 26, 1274-1280.

(9) Dai, S.; Song, H.; Dou, G.; Qian, X.; Zhang, Y.; Cai, Y.; Liu, X.; Tang, Z. Rapid Commun. Mass Spectrom. 2005, 19, 1273-1282. 


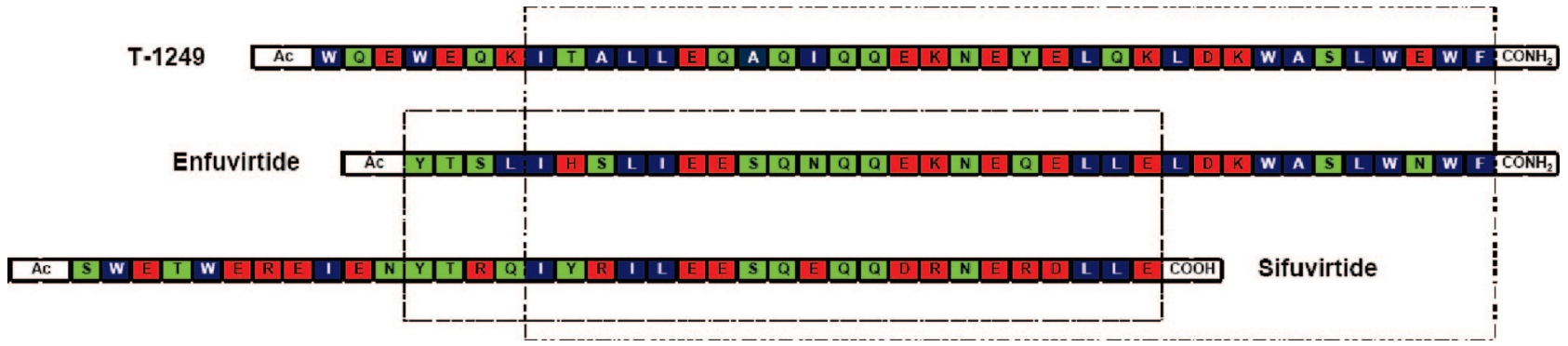

Figure 1. Amino acid sequence of HIV fusion inhibitors T-1249 (top), enfuvirtide (middle), and sifuvirtide (bottom). The boxes indicate a high sequence homology between the peptides. Hydrophobic residues are represented in blue, noncharged polar in green, and charged polar in red.

activity (20× more activity) and improved stability. ${ }^{8,9}$ Clinical trials in the United States are already programmed.

The presence of amphipathic segments, the analysis of the sequence, and the theoretical $\alpha$-helical content ${ }^{10,11}$ and hydrophobicity study ${ }^{12}$ of the amino acid residues suggest that sifuvirtide may interact with biological membranes. The fact that sifuvirtide presents tryptophan residues in its sequence enabled the use of fluorescence spectroscopy techniques ${ }^{13}$ to study its behavior in aqueous solution, its interaction with bio membrane models (large unilammelar vesicles), and its location in phospholipid bilayers.

Previous studies revealed that the fusion inhibitors enfuvirtide and T-1249 interact with membranes in a different way. Enfuvirtide inserts preferentially in fluid-phase lipid membranes ${ }^{14}$ whereas T-1249 may also adsorb on the surface of cholesterol-rich membranes. ${ }^{15}$ The interaction of these peptides with biological membranes may be related to their inhibition efficacy and mode of action, because the binding to gp 41 occurs in an extreme confinement between the cellular membrane and viral envelope. (See ref 16 and references therein).

The aim of this work is to verify the possible interaction of sifuvirtide with lipid membranes, compare it with the results already obtained for enfuvirtide and T-1249, and relate the structural features of these peptides to their different efficiencies to inhibit the HIV-1 fusion process.

\section{Materials and Methods}

2.1. Materials. Sifuvirtide was a kind gift from FusoGen Pharmaceuticals, Inc. 1-Palmitoyl-2-oleoyl-sn-glycero-3-phosphocholine (POPC), 1-palmitoyl-2-oleoyl-sn-glycero-3-ethylphosphocholine (EPOPC), 1,2-dipalmitoyl-sn-glycero-3-phosphocholine (DPPC), 1,2-dipalmitoyl-sn-glycero-3-[phospho-rac-(1-glycerol)] (DPPG, Sodium Salt), and $N$-palmitoyl-D-erythro-sphingosylphosphorylcholine (PSM) were purchased from Avanti Polar-Lipids (Alabaster, AL). 5-Doxylstearic acid (5NS) and 16-doxylstearic acid (16NS) were obtained from Aldrich (Milwaukee, WI). Cholesterol (Chol) was from Sigma (St. Louis, MO), and 1,6-diphenylhexatriene (DPH) as well as trans-parinaric acid ( $\mathrm{tnnA})$ were purchased from

(10) Dwyer, J. J.; Wilson, K. L.; Davison, D. K.; Freel, S. A.; Seedorff, J. E.; Wring, S. A.; Tvermoes, N. A.; Matthews, T. J.; Greenberg, M. L.; Delmedico, M. K. Proc. Natl. Acad. Sci. U.S.A. 2007, 104, 12772-12777.

(11) Bystroff, C.; Shao, Y. Bioinformatics 2002, 18, S54-61.

(12) White, S. H.; Wimley, W. C. Biochim. Biophys. Acta 1998, 1376, 339-352.

(13) Lakowicz, J. R. Principles of Fluorescence Spectroscopy, 2nd ed.; Kluwer Academic/Plenum Publishers: New York, NY, 1999; p 698.

(14) Veiga, S.; Henriques, S.; Santos, N. C.; Castanho, M. Biochem. J. 2004, 377, 107-110.

(15) Veiga, A. S.; Santos, N. C.; Loura, L. M.; Fedorov, A.; Castanho, M. A. J. Am. Chem. Soc. 2004, 126, 14758-14763.

(16) Bar, S.; Alizon, M. J. Virol. 2004, 78, 811-820.
Molecular Probes (Eugene, OR). Trp (L-tryptophan), acrylamide, $N$-2-hydroxyethylpiperazine- $N^{\prime}$-2-ethanesulfonic acid (HEPES), $\mathrm{NaOH}, \mathrm{NaCl}$, chloroform, ethanol, methanol, and dimethylformamide (spectroscopic grade) were obtained from Merck (Darmstadt, Germany).

2.2. Sample Preparation. Sifuvirtide and Trp stock solutions were prepared in $10 \mathrm{mM}$ HEPES buffer, $\mathrm{pH} 7.4,150 \mathrm{mM} \mathrm{NaCl}$. All the experiments were performed at $22{ }^{\circ} \mathrm{C}$, and unless otherwise stated, all solutions were prepared in the same buffer as the stock solutions, which is expected to mimic physiological conditions. Absorption measurements were carried out in a UV-vis spectrophotometer Jasco V-560, and a Malvern Instruments Zetaziser Nano ZS equipped with $\mathrm{He}-\mathrm{Ne}$ laser $(632.8 \mathrm{~nm}, 4.0 \mathrm{~mW})$ was used to perform the dynamic light scattering (DLS) assays. Fluorescence assays were performed in a spectrofluorometer SPEX Fluorolog-3 from Horiba Jobin-Yvon, equipped with a $450 \mathrm{~W}$ Xe lamp (for steady-state fluorescence) and a $279 \mathrm{~nm}$ wavelength-fixed nanosecond light-emitting diode (for time-resolved fluorescence), GlanThompson autopolarizers FL-1044, double monochromators, a single-photon detection module TBX-04-A, and a TCSPC acquisition device DataStation Hub.

2.3. Lipid Preparation. Large unilammelar vesicles (LUV) and small unilammelar vesicles (SUV) were prepared by extrusion and sonication methods, as described elsewhere. ${ }^{17,18}$ LUV were used as models of biological membranes. Several systems were analyzed, including POPC, POPC:EPOPC (9:1, 3:1, and 1:1), POPC:Chol (82:18 and 2:1), DPPC, POPC:DPPC (2:1, 1:1, and 1:2), DPPC: Chol (2:1), POPC:PSM (2:1) and POPC:PSM:Chol (1:1:1) mixtures.

Atomic force microscopy (AFM) measurements were performed to visualize phase separation of the mixtures. These experiments are described in Appendix A (Supporting Information).

2.4. Dynamic Light-Scattering Spectroscopy. For the DLS studies, $800 \mu \mathrm{M}$ stock solutions of sifuvirtide were prepared in filtered HEPES buffer. Successive dilutions of the stock solutions were performed. The samples were filtered with $0.44 \mu \mathrm{m}$ pore nylon membranes before the measurements, and afterward, their concentration was determined by UV-vis absorption measurement at a fixed wavelength of $280 \mathrm{~nm}$.

DLS was performed by using an incident wavelength of 632.8 $\mathrm{nm}$ and a detection angle of $173^{\circ}$ (backscatter detection). Measurements were performed at $25^{\circ} \mathrm{C}$, with an incubation time of $15 \mathrm{~min}$. Data acquisition and analysis was performed by using Malvern DTS Software (www.malvern.co.uk). The autocorrelation curves were analyzed by using the CONTIN method. ${ }^{19,20}$ The average hydrodynamic radius was calculated by considering the dominant population of the intensity distribution of each studied peptide concentration.

2.5. Steady-State Fluorescence Study. Spectral characterization of sifuvirtide and Trp in aqueous solution was performed at an

(17) Mayer, L. D.; Hope, M. J.; Cullis, P. R. Biochim. Biophys. Acta 1986, $858,161-168$

(18) Szoka, F.; Papahadjopoulos, D. Аnпи. Rev. Biophys. Bioeng. 1980, 9 , 467-508.

(19) Provencher, S. W. Comput. Phys. Commun. 1982, 27, 229-242.

(20) Provencher, S. W. Comput. Phys. Commun. 1982, 27, 213-227. 
excitation wavelength of $280 \mathrm{~nm}$, except for the quenching experiments where the excitation was performed at a wavelength of $290 \mathrm{~nm}$ to minimize the relative quencher/fluorophore lightabsorption ratios. For the red-edge excitation shift experiments, ${ }^{21}$ the excitation wavelength varied from 280 to $320 \mathrm{~nm}$. Emission spectra were collected in the 300-450 nm wavelength range, with an integration time of $0.5 \mathrm{~s}$ and an increment of $1 \mathrm{~nm}$. For the quenching experiments, fluorescence was collected at a fixed wavelength of $360 \mathrm{~nm}$. Excitation and emission spectra were corrected for wavelength-dependent instrumental factors. ${ }^{13}$ During the quenching and partition experiments, emission was also corrected for successive dilutions, scatter, ${ }^{22}$ and simultaneous light absorptions of quencher and fluorophore. ${ }^{23}$ The dependence of fluorescence intensity and quantum yield of sifuvirtide ${ }^{24}$ on its concentration was tested over the $0-220 \mu \mathrm{M}$ range, and for these assays, fluorescence intensities were corrected for inner filter effects. ${ }^{25}$ Whenever a constant concentration of peptide was required, $15 \mu \mathrm{M}$ sifuvirtide was used. At this concentration, the absorbance at $280 \mathrm{~nm}$ is below $0.1\left(\varepsilon=1.3 \times 10^{4} \mathrm{M}^{-1} \mathrm{~cm}^{-1}, \ell=\right.$ $0.5 \mathrm{~cm}$ ), ensuring a minimal inner filter effect. Regarding the Trp solutions, a constant concentration of $36 \mu \mathrm{M}$ was used during the experiments. The determination of the relative fluorescence quantum yield $(\Phi)$ of sifuvirtide was performed as indicated in ref 26 , by choosing a Trp solution as standard $(\Phi=0.13)$. Fluorescence anisotropy was used to study red-edge effects of sifuvirtide in aqueous solution (details have been published elsewhere ${ }^{21}$ ). All the data were corrected for background intensities.

2.6. Time-Resolved Fluorescence Study. Fluorescence lifetimes of sifuvirtide and Trp in aqueous solution were calculated from time-resolved intensity decays, which were measured at an excitation wavelength of $279 \mathrm{~nm}$ and an emission acquisition at a wavelength of $360 \mathrm{~nm}$ by using $20 \times 10^{3}$ counts in the peak channel. A $50 \mathrm{~ns}$ range was used for decay acquisition, and channels with less than 200 counts were not used for data analysis. Instrumental response functions were generated from scatter dispersion (glycogen solution (Acros Organics, Belgium)). Data analysis was carried out by using a nonlinear least-squares iterative convolution method based on the Marquardt algorithm. ${ }^{27}$ The goodness of the fit was judged from the global $\chi^{2}$ values, distribution of the residuals, and autocorrelation plot. The fluorescence decay was complex and described by the sum of two exponentials. ${ }^{13}$

Sifuvirtide and Trp intensity decays were corrected for background intensities and scatter. The average lifetime $(\langle\tau\rangle)$ and the fluorescence lifetime averaged by the pre-exponential factors $(\bar{\tau})$ were recovered (details have been published elsewhere ${ }^{21}$ ).

2.7. Quenching Experiments. Fluorescence quenching assays of sifuvirtide by acrylamide, $5 \mathrm{NS}$, and $16 \mathrm{NS}$ were performed by both steady-state and time-resolved fluorescence spectroscopy. Quenching of $15 \mu \mathrm{M}$ sifuvirtide by acrylamide $(0-60 \mathrm{mM})$ was studied in buffer and in the presence of $3 \mathrm{mM}$ of lipid (LUV), by successive additions of small volumes. The quenching assays by the lipophilic probes $5 \mathrm{NS}$ and $16 \mathrm{NS}$ were carried out at the same peptide and lipid concentrations, by successive additions of small amounts of these quenchers to samples of peptide incubated in LUV, keeping the ethanol concentration below $2 \%(\mathrm{v} / \mathrm{v}){ }^{28}$ The effective lipophilic quencher concentration in the membrane was calculated from the partition coefficient of both quenchers to the lipid

(21) Santos, N. C.; Castanho, M. Trends Appl. Spectrosc. 2002, 4, 113 125.

(22) Ladokhin, A. S.; Jayasinghe, S.; White, S. H. Anal. Biochem. 2000, 285, 235-245.

(23) Coutinho, A.; Prieto, M. J. Chem. Educ. 1993, 70, 425-428.

(24) Henriques, S. T.; Castanho, M. A. Biochim. Biophys. Acta 2005, 1669, $75-86$.

(25) Kubista, M.; Sjoback, R.; Eriksson, S.; Albinsson, B. Analyst 1994, $119,417-419$.

(26) Fery-Forgues, S.; Lavabre, D. J. Chem. Educ. 1999, 76, 1260-1264.

(27) Marquardt, D. W. SIAM J. Appl. Math. 1963, 11, 431-441.

(28) Yamazaki, M.; Miyazu, M.; Asano, T.; Yuba, A.; Kume, N. Biophys. J. 1994, 66, 729-733. bilayers. ${ }^{29}$ For every addition, a minimal 10 min incubation time was allowed before measurement.

Quenching data were analyzed by using the Stern-Volmer equation $^{21}$ (eq 1) or, when a negative deviation to the Stern-Volmer relationship was observed, by using eq $2 .^{29,30}$

$$
\frac{I_{0}}{I}=\frac{\frac{I_{0}}{I}=1+K_{\mathrm{SV}}[\mathrm{Q}]}{1+K_{\mathrm{SV}}[\mathrm{Q}]}
$$

where $I$ and $I_{0}$ are the fluorescence intensity of the sample in the presence and absence of quencher, respectively, $K_{\mathrm{SV}}$ is the Stern-Volmer constant, $[Q]$ the concentration of quencher, and $f_{\mathrm{B}}$ is the fraction of light accessible to the quencher.

In the case of dynamic quenching, the relationship $I_{0} / I=\bar{\tau}_{0} / \tau$ is valid; thus, time-resolved quenching data can be analyzed by using eqs 1 and 2 . Bimolecular rate constants $\left(k_{\mathrm{q}}\right)$ were calculated according to ref 21 .

2.8. Extent of Partition to LUV. Membrane partition studies were performed by successive additions of small amounts of a 15 $\mathrm{mM}$ lipid suspension (LUV) to a $15 \mu \mathrm{M}$ sifuvirtide solution, with a 10 min incubation time in between each addition. The extent of the interaction of the peptide with the modeled lipid system was judged by steady-state and time-resolved fluorescence.

The partition coefficients $\left(K_{\mathrm{p}}\right)$ were calculated from the fit of the experimental data with eq $3 .^{31}$

$$
\frac{I}{I_{\mathrm{W}}}=\frac{1+K_{\mathrm{p}} \gamma_{\mathrm{L}} \frac{I_{\mathrm{L}}}{I_{\mathrm{W}}}[\mathrm{L}]}{1+K_{\mathrm{p}} \gamma_{\mathrm{L}}[\mathrm{L}]}
$$

where $I_{\mathrm{W}}$ and $I_{\mathrm{L}}$ are the fluorescence intensities in aqueous solution and in lipid, respectively, $\gamma_{\mathrm{L}}$ is the molar volume of the lipid,,$^{32,33}$ and $[L]$ is the lipidic concentration.

In addition to the fluorescence intensities $(I)$, the fluorescence lifetimes averaged by the pre-exponential factors $(\bar{\tau})$ could also be used in eq 3 during the assays. ${ }^{31}$

The fraction of peptide interacting with membranes $\left(x_{\mathrm{L}}\right)$ and the fraction of light emitted by the peptide incorporated in the vesicles $\left(f_{\mathrm{L}}\right)$ were determined according to refs 24 and 29.

2.9. Membrane Fluidity Perturbation Assay. To judge the influence of the peptide sifuvirtide on the fluidity of gel-phase DPPC membranes, the steady-state anisotropy of tPnA (calculated according to ref 21) was studied at a fixed concentration of lipid (3 $\mathrm{mM})$ as a function of the peptide concentration $(0-50 \mu \mathrm{M})$. A total of $5 \mu \mathrm{M}$ of a solution of tPnA in ethanol was added into a $3 \mathrm{mM}$ lipid suspension of DPPC LUV (1:600 dilution) and was incubated over $30 \mathrm{~min}$ at a temperature above that of the phase transition. ${ }^{34}$ Ethanol concentration in the sample was kept below $2 \%(\mathrm{v} / \mathrm{v}){ }^{28}$ Excitation of tPnA was performed at a wavelength of $318.5 \mathrm{~nm}$, coinciding with the relative absorption maximum presented by this probe in DPPC LUV. Emission spectra were collected over the $325-550 \mathrm{~nm}$ range, and the fluorescence intensities were corrected for the dilution effects.

2.10. Förster Resonance Energy Transfer (FRET) Experiment. The evaluation of possible adsorption of sifuvirtide on the gel-phase DPPC membranes was performed by a FRET study, by using the Trp residues of the peptide as donors and DPH molecules

(29) Santos, N. C.; Prieto, M.; Castanho, M. A. Biochemistry 1998, 37, 8674-8682.

(30) Lehrer, S. S. Biochemistry 1971, 10, 3254-63.

(31) Santos, N. C.; Prieto, M.; Castanho, M. A. Biochim. Biophys. Acta 2003, 1612, 123-135.

(32) Chiu, S. W.; Jakobsson, E.; Subramaniam, S.; Scott, H. L. Biophys. J. 1999, 77, 2462-2469.

(33) Nagle, J. F.; Wiener, M. C. Biochim. Biophys. Acta 1988, 942, 1-10.

(34) Castanho, M.; Prieto, M.; Acuna, A. U. Biochim. Biophys. Acta 1996, 1279, 164-168. 
as acceptor. ${ }^{35}$ Small amounts of DPH in dimethylformamide (DMF) were added into a DPPC suspension, keeping the DMF concentration below $1 \%(\mathrm{v} / \mathrm{v})$. To guarantee a complete incorporation of $\mathrm{DPH}$, it was incubated for $12 \mathrm{~h}$ with DPPC vesicle suspensions at $4{ }^{\circ} \mathrm{C}$. A concentration range of $0-60 \mu \mathrm{M}$ of DPH in the membranes $(0-2 \%$ over the total lipid concentration) was studied. After the complete incorporation of DPH, peptide was added to the lipid suspension, and the solution was kept incubating during 10 min at room temperature. The lipid and peptide final concentrations used in this assay were $3 \mathrm{mM}$ and $15 \mu \mathrm{M}$, respectively. Time-resolved fluorescence was used to evaluate the FRET. Control measurements of the DPH/DPPC lipid suspensions without peptide were performed to discard any background intensity and scatter contribution.

Energy-transfer efficiencies, $E$, were experimentally determined from eq 4.

$$
E=1-\frac{\bar{\tau}_{\mathrm{DA}}}{\bar{\tau}_{\mathrm{D}}}
$$

where $\bar{\tau}_{\mathrm{DA}}$ and $\bar{\tau}_{\mathrm{D}}$ are the fluorescence lifetimes averaged by the pre-exponential factors in the presence and absence of acceptor, respectively.

The theoretical Förster radius $\left(R_{0}\right.$, critical distance for energy transfer) was calculated according to Berberan-Santos and Prieto. ${ }^{36}$

$$
R_{0}=0.2108\left[\kappa^{2} \Phi_{\mathrm{D}} n^{-4} \int_{0}^{\infty} I(\lambda) \varepsilon(\lambda) \lambda^{4} \mathrm{~d} \lambda\right]^{1 / 6}
$$

where $\kappa^{2}$ is the orientation factor (the value $\kappa^{2}=2 / 3$, relative to the dynamic isotropic limit, was used in this study), $\Phi_{\mathrm{D}}$ is the donor quantum yield in the absence of acceptor (the value $\Phi_{\mathrm{D}}=0.08$, measured in this study for $3 \mathrm{mM}$ DPPC lipid concentration, was used), $n$ is the refractive index ( $n=1.4$ was used in this study as refractive index within the vesicles $\left.{ }^{37}\right), I(\lambda)$ is the normalized donor emission spectrum, and $\varepsilon(\lambda)$ is the acceptor molar absorption spectrum (expressed in $\mathrm{M}^{-1} \mathrm{~cm}^{-1}$ ). If in eq $5 \lambda$ is in nanometers, the calculated $R_{0}$ will be in angstroms.

The FRET formalism, ${ }^{15,37}$ proposed here to evaluate an adsorption phenomenon, assumes two planes: an acceptor plane at the center of the DPPC bilayer and a donor plane at a distance $W$ from the acceptor plane. In this context, the fluorescence decay law of the donor in the presence of an acceptor $\left(I_{\mathrm{D}, \mathrm{A}}\right)$ is given by

$$
I_{D, A}=A_{\mathrm{W}} I_{\mathrm{D}, \mathrm{W}}(t)+\left(1-A_{\mathrm{W}}\right) I_{\mathrm{D}, \mathrm{L}}(t) \rho(t)
$$

where $A_{\mathrm{W}}$ is the fraction of the molecules in aqueous solution, $I_{\mathrm{D}, \mathrm{W}}$ and $I_{\mathrm{D}, \mathrm{L}}$ are the fluorescence decays of the donor in the buffer and adsorbed to the lipid vesicles, respectively, and $\rho(t)$ is the function that denotes the FRET rate to acceptors at an interplanar plane distance of $W$ away from the donor. This quantity is given by

$$
\begin{aligned}
\rho(t)=\exp \left\{-2\left(\frac{W}{R_{0}}\right)^{2} \frac{[\mathrm{DPH}]}{[\mathrm{DPPC}] a_{\mathrm{DPPC}}} \times\right. & \\
& \left.\pi R_{0}^{2} \int_{0}^{1} \frac{1-\exp \left[\left(-\frac{t}{\langle\tau\rangle}\right)\left(\frac{R_{0}}{W}\right)^{6} \alpha^{6}\right]}{\alpha^{3}} \mathrm{~d} \alpha\right\}
\end{aligned}
$$

where $\langle\tau\rangle$ is the average lifetime of the donor, $R_{0}$ is the Förster radius, $[\mathrm{DPH}]$ is the concentration of $\mathrm{DPH}$, and $a_{\mathrm{DPPC}}$ is the area per DPPC molecule. ${ }^{38}$ In the absence of acceptor, $\rho(t)=0$.

(35) Le Doan, T.; Takasugi, M.; Aragon, I.; Boudet, G.; MontenayGarestier, T.; Helene, C. Biochim. Biophys. Acta 1983, 735, 259270 .

(36) Berberan-Santos, M. N.; Prieto, M. J. E. J. Chem. Soc., Faraday Trans. 1987, 83, 1391-1410.

(37) Davenport, L.; Dale, R. E.; Bisby, R. H.; Cundall, R. B. Biochemistry 1985, 24, 4097-4108.

(38) Marsh, D. CRC Handbook of Lipid Bilayers; CRC Press: Boston, 1990.
The experimental energy-transfer efficiencies were adjusted to this formalism by calculating the expected energy-transfer efficiencies by numerical integration of the theoretical decay laws of the donor in the presence and absence of acceptor, recovering the values of $W, A_{\mathrm{W}}$, and $R_{0}$.

\section{Results and Discussion}

3.1. Characterization of Sifuvirtide in Aqueous Solution. Spectra of peptides presenting aromatic residues can give valuable information about local structural properties. ${ }^{13,21}$ Fluorescence spectral characteristics of sifuvirtide are dominated by its two Trp residues (Figure 2A). In solution and under physiological $\mathrm{pH}$ and ionic strength conditions, sifuvirtide (15 $\mu \mathrm{M})$ has an absorption maximum at $280 \mathrm{~nm}$ and an emission maximum at $347 \mathrm{~nm}$. No red-edge excitation-shift effects were detected under these conditions. The fluorescence decay of the peptide was described by the sum of two exponentials with a mean lifetime of $\tau=2.45 \mathrm{~ns}\left(\chi^{2} \leq 1.3\right)$. A value of $\Phi=0.07$ was recovered for the fluorescence quantum yield $(\Phi)$ of the peptide.

Fluorescence quenching of the Trp residues of the peptide by acrylamide was also studied to verify the accessibility of the fluorophores. The quenching was evaluated by steady-state and time-resolved fluorescence to distinguish static from dynamic quenching behaviors. ${ }^{13,21}$ A predominance of dynamic quenching of the fluorescence of sifuvirtide by acrylamide was observed (Figure 2B). Linear Stern-Volmer plots were obtained (bimolecular rate constant $\left.k_{\mathrm{q}}=(3.26 \pm 0.03) \times 10^{-9} \mathrm{M}^{-1} \mathrm{~s}^{-1}\right)$, revealing that the Trp residues are not localized inside hydrophobic pockets and are accessible to the quencher.

All these results suggest that the Trp residues of sifuvirtide are considerably exposed to the surrounding aqueous medium. ${ }^{13,21}$

Furthermore, to evaluate possible changes in the supramolecular structure of sifuvirtide, the fluorescence quantum yield dependence and the hydrodynamic radius dependence on peptide concentration were studied. A concentration-dependent behavior is often related to different structures of the peptide because of folding variation or oligomerization. ${ }^{24}$ Regarding fluorescence results, no significant deviations from linearity were observed at concentrations less than $60 \mu \mathrm{M}$ (Figure 2C). A monomeric form of the peptide could then be assumed in this situation. At higher concentrations, a positive deviation from linearity was observed and could indicate the formation of small aggregates of peptide, where Trp residues are localized in a less-polar environment, presenting a higher quantum yield. ${ }^{24}$ Because lightscattering techniques have a very low sensitivity for small aggregates, ${ }^{39}$ during the dynamic light-scattering assays (Figure 2D), no significant aggregation until a concentration of $600 \mu \mathrm{M}$ (near the solubility limit) was revealed.

\subsection{Interaction of Sifuvirtide with Biological Membrane} Model Systems.

3.2.1. Extent of Partition into Zwitterionic Lipid Bilayers. The partition of sifuvirtide into zwitterionic lipid bilayers was evaluated to study the importance of the lipidic phase in this interaction. Previous studies revealed that HIV fusion inhibitors may interact with lipid membranes, ${ }^{14,15}$ which is regarded as relevant for their mode of action, because the inhibition process of this class of peptides occurs in extreme confinement between the cellular plasmatic membrane and the viral envelope. (See ref 16 and references therein). Several vesicle systems of varying lipid composition were analyzed to mimic the different rigidity

(39) Banachowicz, E. Biochim. Biophys. Acta 2006, 1764, 405-413. 

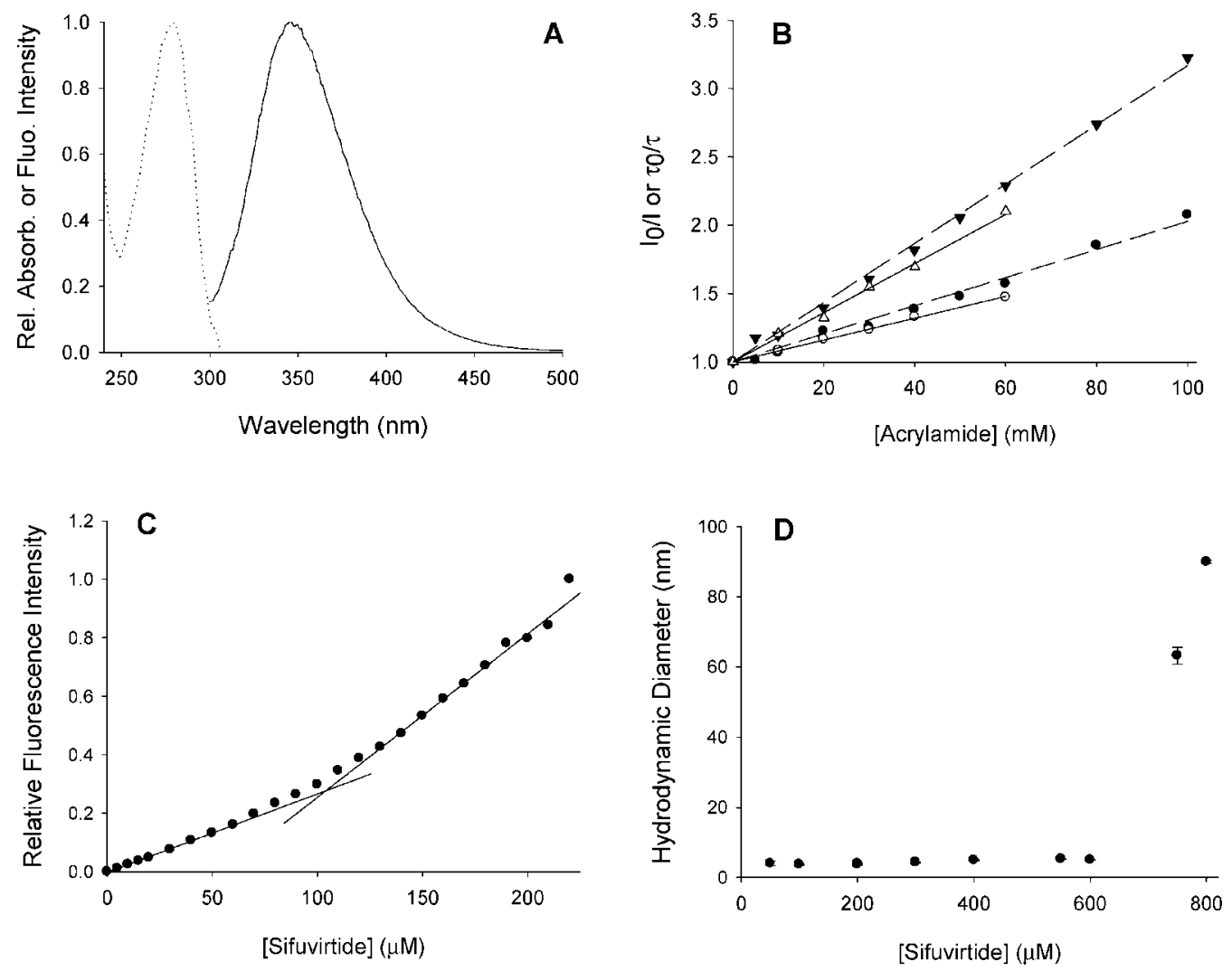

Figure 2. Characterization of sifuvirtide in aqueous solution. (A) Absorption (dotted line) and fluorescence emission (solid line) spectra of $15 \mu \mathrm{M}$ sifuvirtide. Emission was induced by exciting sifuvirtide at a wavelength of $280 \mathrm{~nm}$. (B) Quenching of the Trp residues of $15 \mu \mathrm{M}$ sifuvirtide (circles) and $36 \mu \mathrm{M}$ free Trp in solution (triangles) by the soluble quencher acrylamide by using steady-state (solid symbols) and time-resolved (open symbols) fluorescence. Dashed and solid lines are fittings of eq 1 to the experimental data. (C) Fluorescence intensity dependence on sifuvirtide concentration. A positive deviation of linearity was detected. (D) Hydrodynamic radius dependence on sifuvirtide concentration, studied by dynamic light-scattering spectroscopy. Each point corresponds to an average of 5-13 measurements. Error bars represent the standard deviation. All the assays were performed in a $10 \mathrm{mM}$ HEPES buffer, $\mathrm{pH} 7.4$, containing $150 \mathrm{mM} \mathrm{NaCl}$.

properties more common at the cellular level. AFM was used to visualize the existence of the phase separation in several of those mixtures (Appendix A, Supporting Information). Titrations of peptide $(15 \mu \mathrm{M})$ were performed at room temperature with POPC vesicles (liquid-disordered phase), DPPC or PSMcontaining vesicles (gel phase), and vesicles containing cholesterol in their composition (liquid-ordered phase). No significant changes in fluorescence intensity nor spectral blue-shifts were observed when sifuvirtide was in the presence of POPC, POPC:Chol (2:1), POPC:PSM:Chol (1:1:1), or DPPC:Chol (2: 1) vesicles. Only in the presence of pure DPPC gel-phase membranes, a significant increase in the fluorescence intensity (Figure 3A) and quantum yield, as well as a significant spectral blue-shift (7 nm), were observed (data not shown). A specific and preferential interaction of sifuvirtide with the more-rigid membranes seems to occur, and the increase of fluidity reduces this interaction (as seen with DPPC:Chol or POPC:DPPC vesicles). Increased fluorescence lifetimes of sifuvirtide were also obtained in the presence of gel-phase membranes (data not shown).

No significant alterations of the fluorescence mean lifetime of sifuvirtide in the presence of homogeneous liquid-ordered and liquid-disordered membranes were observed. Nevertheless, when the peptide was interacting with POPC:DPPC vesicles (data not shown), the increase in the DPPC content led to an increase of the mean lifetime of the fluorophore. This suggests that the interaction of sifuvirtide with biomembranes depends preferentially on the presence of more rigid membrane regions.
3.2.2. Extent of Partition into Charged Lipid Bilayers. Vesicles with cationic and anionic phospholipids were used as positive and negative controls of partition.

The addition of EPOPC, a fluid-phase (at room temperature) cationic (at $\mathrm{pH}$ 7.4) phospholipid, into POPC vesicles forced the partition of anionic sifuvirtide into the membranes. Large spectral shifts (data not shown) and an increase in the fluorescence intensity (Figure 3A), quantum yield (data not shown), and lifetime (data not shown) were observed. A partition coefficient of $K_{\mathrm{p}}=(2.2 \pm 0.3) \times 10^{3}$ was recovered for the interaction with POPC:EPOPC $(1: 1)$ by using eq 3 . Thus, the use of EPOPC in the vesicles served as positive control for the interaction with fluid-phase membranes, discarding the hypothesis that interaction with POPC could occur without being reported by Trp fluorescence because of any kind of shielding.

DPPG vesicles were used as negative control for the interaction of sifuvirtide with DPPC gel-phase membranes. The repulsion between the negatively charged glycerol headgroup and sifuvirtide prevents the peptide from interacting with gelphase vesicles. Thus, at variance with the interaction between sifuvirtide and DPPC vesicles, no interaction was observed with DPPG vesicles (Figure 3A).

3.2.3. Quenching of Sifuvirtide with Acrylamide in the Presence of Lipid Vesicles. Acrylamide is a soluble quencher in aqueous solution with low capacity for penetration into lipid bilayers. ${ }^{40}$ It is generally utilized to verify the aqueous environment accessibility of the Trp residues in the peptide structure ${ }^{24,41,42}$ and infer on the depth of interaction between peptides and lipid 

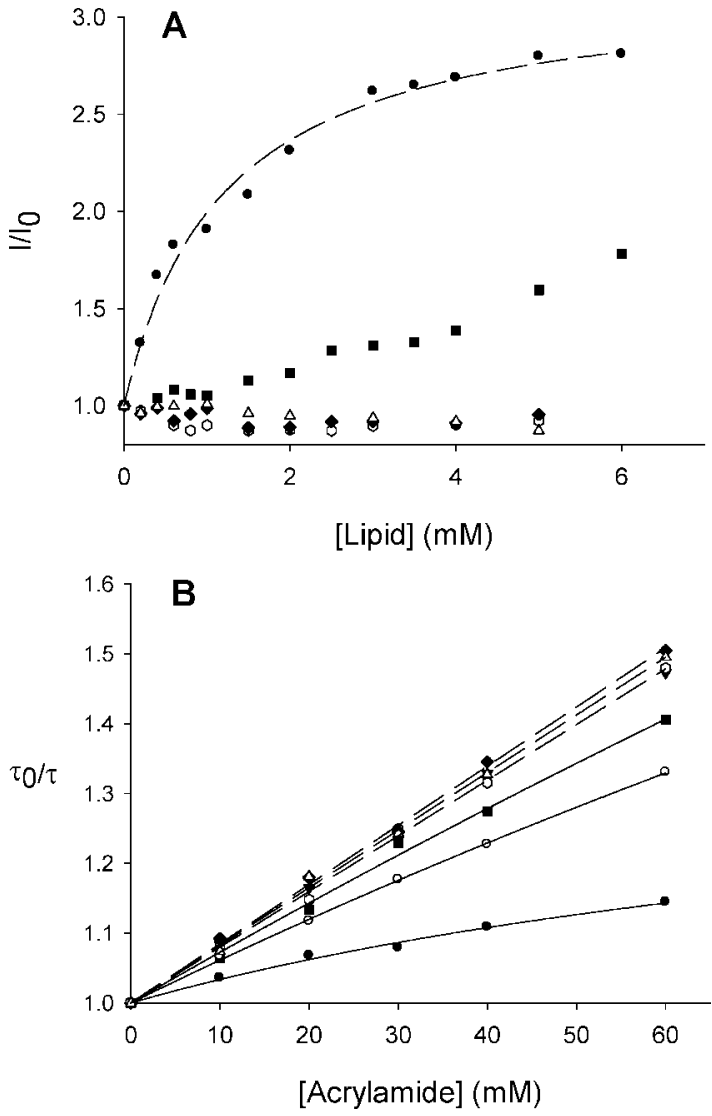

Figure 3. Interaction of sifuvirtide with lipid membranes. (A) Partition of $15 \mu \mathrm{M}$ sifuvirtide into large unilamellar vesicles performed by successive additions of a concentrated LUV suspension. (B) Fluorescence quenching of $15 \mu \mathrm{M}$ sifuvirtide by acrylamide in the presence of $3 \mathrm{mM}$ lipid vesicles. All the assays were performed in a $10 \mathrm{mM}$ HEPES buffer, $\mathrm{pH}$ 7.4, containing $150 \mathrm{mM} \mathrm{NaCl}$. The line is a fitting of eq 3 to the experimental data. Studied systems: aqueous solution (solid triangles); POPC (hexagons); DPPC (squares); POPC:Chol (diamonds); DPPG (open triangles); POPC:EPOPC (3:1, open circles); and POPC:EPOPC (1:1, solid circles).

bilayers. ${ }^{24,41}$ By interacting with membranes, the peptide is less accessible to the quencher in solution, and therefore, the fluorescence intensity and lifetime will be less quenched. A characteristic negative deviation in the Stern-Volmer relationship (eq 1) can then be observed, and by fitting the Lehrer equation (eq 2) to the data, it is possible to obtain the fraction of peptide that is buried in the lipid bilayers. Therefore, the use of acrylamide in this context complements the partition assays previously described.

Quenching Stern-Volmer plots in the presence of $3 \mathrm{mM}$ of lipid and $15 \mu \mathrm{M}$ of peptide revealed a linear profile, similar to the quenching profile observed for sifuvirtide in solution, for almost all the vesicle systems analyzed. The exceptions were DPPC vesicles and POPC:EPOPC vesicles (Figure 3B). In these cases, negative deviations to the Stern-Volmer relationship were observed, indicating the presence of a fraction of peptide that is interacting with the vesicles and is consequently less accessible to the quencher. By fitting the Lehrer equation (eq 2) to the data, the values of the emission intensity fraction accessible to the quencher, $f_{\mathrm{B}}=0.84$ and $f_{\mathrm{B}}=0.29$, were

(40) Moro, F.; Goni, F. M.; Urbaneja, M. A. FEBS Lett. 1993, 330, 129132.

(41) Melo, M. N.; Castanho, M. A. Biochim. Biophys. Acta 2007, 1768, $1277-1290$.

(42) Veiga, A. S.; Castanho, M. A. FEBS J. 2007, 274, 5096-50104.
Table 1. Parameters Obtained for the Quenching of the Fluorescence of Sifuvirtide by Acrylamide and by the Lipophilic Probes 5NS and $16 \mathrm{NS}^{a}$

\begin{tabular}{|c|c|c|c|c|}
\hline & \multicolumn{2}{|c|}{ POPC:EPOPC (1:1) } & \multicolumn{2}{|c|}{ DPPC } \\
\hline & steady state & time resolved & steady state & time resolved \\
\hline$K_{\mathrm{SV}, \text { acryl }}\left(\mathrm{M}^{-1}\right)$ & & $12.5 \pm 3.10$ & & $8.80 \pm 2.00$ \\
\hline$f_{\mathrm{B}, \mathrm{acryl}}$ & & $0.29 \pm 0.16$ & & $0.84 \pm 1.06$ \\
\hline$f_{\mathrm{L}, \text { acryl }}$ & & $0.71 \pm 0.40$ & & $0.16 \pm 0.21$ \\
\hline$K_{\mathrm{SV}, 5 \mathrm{NS}}\left(\mathrm{M}^{-1}\right)$ & $6.70 \pm 0.80$ & $1.90 \pm 0.06$ & $6.94 \pm 2.70$ & $12.6 \pm 6.30$ \\
\hline$K_{\mathrm{SV}, 16 \mathrm{NS}}\left(\mathrm{M}^{-1}\right)$ & $2.20 \pm 0.40$ & $2.40 \pm 0.07$ & $21.3 \pm 10.2$ & $12.5 \pm 2.30$ \\
\hline$f_{\mathrm{B}, 5 \mathrm{NS}}$ & $0.79 \pm 0.32$ & 1 & $0.29 \pm 0.24$ & $0.14 \pm 0.15$ \\
\hline$f_{\mathrm{B}, 16 \mathrm{NS}}$ & $0.86 \pm 0.73$ & 1 & $0.12 \pm 0.12$ & $0.15 \pm 0.06$ \\
\hline
\end{tabular}

${ }^{a}$ Assays were performed in a $10 \mathrm{mM}$ HEPES buffer, $\mathrm{pH}$ 7.4, containing $150 \mathrm{mM} \mathrm{NaCl}$. Quenching experiments were performed at a total lipidic concentration of $3 \mathrm{mM}$. $K_{\mathrm{SV}}$ and $f_{\mathrm{B}}$ were determined by fitting eq 2 or eq $1\left(f_{\mathrm{B}}=1\right)$ to the experimental data. $f_{\mathrm{L} \text {,acryl }}$ was obtained by $f_{\mathrm{L}}=1-f_{\mathrm{B}}$.

recovered in the presence of DPPC and POPC:EPOPC (1:1) vesicles, respectively. Considering that acrylamide has a low affinity to the lipid, the values of the fraction of fluorescence intensity originating from the peptide in the lipid $\left(f_{\mathrm{L}}\right)$ can then be obtained, $f_{\mathrm{L}}=1-f_{\mathrm{B}}$ (Table 1$)$.

3.2.4. Localization of Sifuvirtide in Lipid Bilayers. A differential quenching approach by combining two lipophilic Trp quenchers enables one to determine the distribution of the location of the Trp residues of sifuvirtide in the lipid bilayers. ${ }^{43}$ The probes $5 \mathrm{NS}$ and $16 \mathrm{NS}$ were used as quenchers. The efficiency of quenching depends on the proximity of the Trp residues of the peptide to the quenchers. Considering their distributions, $5 \mathrm{NS}$ probes essentially the interface of the bilayers, whereas $16 \mathrm{NS}$ probes its core.

As expected, no changes in the fluorescence intensity and lifetime were detected in the presence of 5NS and 16NS in POPC or POPC:Chol vesicles. This result is further evidence of nonsignificant interaction of the peptide with fluid-phase and cholesterol-enriched membranes (liquid-ordered phase). Nevertheless, in the presence of DPPC and POPC:EPOPC (3:1 and 1:1) LUVs, the fluorescence of the Trp residues of sifuvirtide was quenched by $5 \mathrm{NS}$ and $16 \mathrm{NS}$. A negative deviation to the Stern-Volmer relationship was recovered (Figure 4A), indicating the presence of two subpopulations of fluorophore relatively to the accessibility to the quenchers. The Stern-Volmer constants $\left(K_{\mathrm{SV}}\right)$ and the fraction of peptide accessible to the quenchers $\left(f_{\mathrm{B}}\right)$ were recovered by using eqs 1 and 2 and are presented in Table 1.

Steady-state fluorescence revealed that the peptide was more efficiently quenched by $5 \mathrm{NS}$, indicating a preferred location near the lipid bilayer interface. Time-resolved fluorescence was utilized to evaluate the predominance of static or dynamic quenching effects ${ }^{13}$ by $5 \mathrm{NS}$ and 16NS. Dynamic quenching was predominant; but for $5 \mathrm{NS}$, a strong static contribution was detected, especially in the POPC:EPOPC vesicles. This elevated static contribution is due to the close proximity of the fluorophore to the 5NS quencher. It is also interesting that the quenching efficiency is increased (higher $K_{\mathrm{SV}}$ ) in gel-phase membranes. This can be caused by a segregation effect of both quencher and peptide to defect lines on the gel membrane, enabling a local concentration that favors efficiency enhancement.

The $f_{\mathrm{B}}$ values obtained by applying the Lehrer equation (eq 2 ) to the $5 \mathrm{NS}$ and $16 \mathrm{NS}$ quenching experimental data can be

(43) Fernandes, M. X.; Garcia de la Torre, J.; Castanho, M. A. Anal. Biochem. 2002, 307, 1-12. 

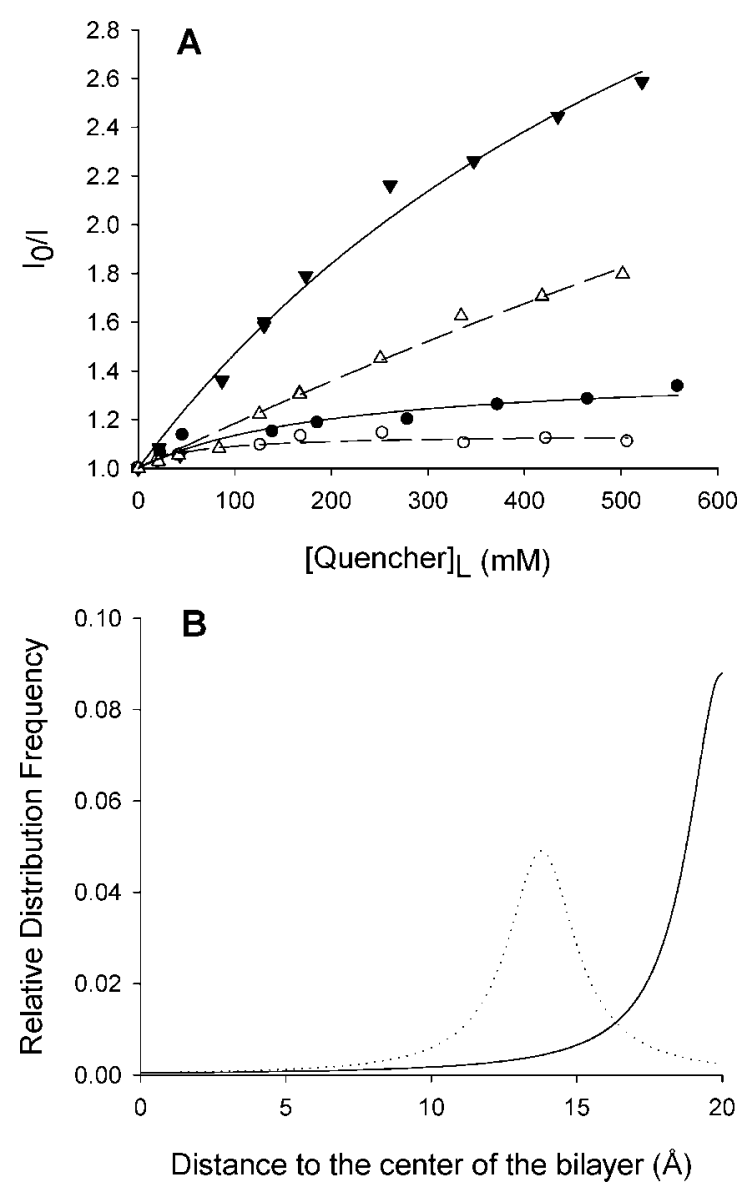

Figure 4. Location of $15 \mu \mathrm{M}$ sifuvirtide in lipid bilayers $(3 \mathrm{mM})$. (A) Stern-Volmer plot of the quenching of $15 \mu \mathrm{M}$ sifuvirtide by $5 \mathrm{NS}$ (solid symbols) and 16NS (open symbols) in POPC:EPOPC (1:1) (triangles) and DPPC (circles) by using steady-state fluorescence spectroscopy. Dashed and solid lines are fittings of eq 2 to the experimental data. (B) In-depth distribution of the Trp residues of sifuvirtide in lipid bilayers by using the SIMEXDA method. The location is mainly interfacial for POPC:EPOPC (1:1) vesicles (dashed line) and superficial for DPPC vesicles (solid line).

compared to the $f_{\mathrm{L}}$ values obtained for the partition of sifuvirtide into the different vesicle systems ${ }^{24}$ and to $f_{\mathrm{L}}$ values obtained for the fluorescence quenching with acrylamide. As seen in Table 1, given the intrinsic experimental error, $f_{\mathrm{B}, 5 \mathrm{NS}}$ and $f_{\mathrm{B}, 16 \mathrm{NS}}$ are in agreement with $f_{\mathrm{L} \text {,acryl }}$ for the interaction of sifuvirtide with DPPC and POPC:EPOPC vesicles.

An improved version of the SIMEXDA method ${ }^{43}$ that takes into account the possibility of static quenching by using a sphere-of-action methodology ${ }^{44}$ was used to obtain the in-depth distribution of the Trp residues of sifuvirtide in DPPC and POPC:EPOPC (1:1) vesicles. Regarding the interaction with gelphase DPPC vesicles (Figure 4B), a superficial location of the Trp residues of sifuvirtide was observed. As the maximum of the distribution coincides with the top of the bilayer, an adsorption of sifuvirtide on these rigid membranes is suggested, but insertion to a certain extent cannot be yet discarded. In contrast, for the electrostatic interaction of the peptide with POPC:EPOPC (1:1), the distribution of the Trp residues suggests an insertion in the bilayer (Figure 4B).

3.2.5. Adsorption of Sifuvirtide on Gel Phase Membranes. The preference of sifuvirtide for gel-phase membranes is quite

(44) Castanho, M. A.; Prieto, M. J. Biochim. Biophys. Acta 1998, 1373, $1-16$. meaningful and was further studied. The quenching and partition studies suggest a superficial location of sifuvirtide and a low extent of partitioning to the gel-phase membranes. An adsorption interaction can be hypothesized.

tPnA is a fluorescent tetraene fatty acid with partitions preferentially into gel-phase membranes, ${ }^{34,45}$ commonly used as a probe in phase-transition studies. ${ }^{46}$ If the peptide inserts into membranes, a pertubation in the anisotropy value of tPnA should be detected because of the fluidification of the membrane caused by this insertion. As the peptide sifuvirtide was added to a suspension of DPPC vesicles (3 mM) with 1:600 M of tPnA, no changes in the average anisotropy of tPnA were detected (data not shown), showing that the interaction of the peptide does not lead to a significant perturbation of the membrane fluidity. These results are consistent with a superficial interaction of the peptide with gel-phase membranes and in agreement with the adsorption hypothesis.

To confirm the adsorption, a FRET experiment was conducted by using the Trp residues of sifuvirtide as donor and DPH as acceptor. DPH is a rigid rodlike fluorescent probe that is a suitable acceptor to $\operatorname{Trp}^{35}$ because of the overlapping of its absorption spectrum with the emission spectrum of Trp. This probe partitions deeply within the core of the bilayer. Molecular dynamics studies reveal that DPH prefers to be accommodated in the hydrophobic acyl chain region of DPPC, oriented with its long axis parallel to the bilayer normal. ${ }^{47}$ For the sake of simplicity, a commonly used distribution of DPH at the center of the bilayer can be considered as a reasonable crude approximation, ${ }^{37}$ also given that the DPH molecules will distribute symmetrically between the two bilayer leaflets.

Energy transfer was followed by time-resolved fluorescence in the presence of $3 \mathrm{mM}$ of DPPC, $15 \mu \mathrm{M}$ of sifuvirtide, and a variable concentration of DPH $(0-2 \%)$. An increase in the energy-transfer efficiency with the DPH content was observed (Figure 5A). The existence of FRET is a direct evidence of an interaction of sifuvirtide with DPPC gel-phase membranes. By calculating the energy-transfer efficiencies through numerical integration of the theoretical decay laws (eqs 6 and 7 ), not only the interplanar distance $(W)$ can be recovered but also the Förster radius $\left(R_{0}\right)$ and the fraction of peptide in solution $\left(A_{\mathrm{W}}\right)$ (and consequently the adsorption coefficient $\left.\left(K_{\mathrm{a}}\right)\right)$. A Förster radius of $29.9 \AA$ was obtained by using this methodology, which is in agreement with the Förster radius obtained (30.6 $\AA$ ) for the Trp-DPH donor-acceptor pair ${ }^{36}$ (eq 5). We have estimated an interplanar distance of $32.4 \AA$ between DPH (at the center of the bilayer) and the Trp residues of sifuvirtide. Sifuvirtide adopting an $\alpha$-helical secondary structure is expected to have a maximum diameter of $15 \AA$ (calculated by using PyMOL, http://www.pymol.org). Assuming a $44 \AA$ length for a gel-phase DPPC bilayer, ${ }^{38} 22$ $\AA<W<37 \AA$ is estimated, depending on the orientation of the Trp residues. The interplanar distance experimentally obtained ( $W=32.4 \AA$ ) lies in this range and, therefore, is in agreement with the adsorption (Figure 5B). A hypothetical value of $A_{\mathrm{W}}=0.75$ was obtained; thus, $K_{\mathrm{a}}=0.16 \times 10^{3}$ is retrieved, indicating a reasonable degree of adsorption of sifuvirtide on the surface of the gel-phase membranes.

(45) Sklar, L. A. Mol. Cell. Biochem. 1980, 32, 169-77.

(46) de Almeida, R. F.; Loura, L. M.; Fedorov, A.; Prieto, M. Biophys. J. 2002, 82, 823-834.

(47) Repakova, J.; Capkova, P.; Holopainen, J. M.; Vattulainen, I. J. Phys. Chem. B 2004, 108, 13438-13448. 


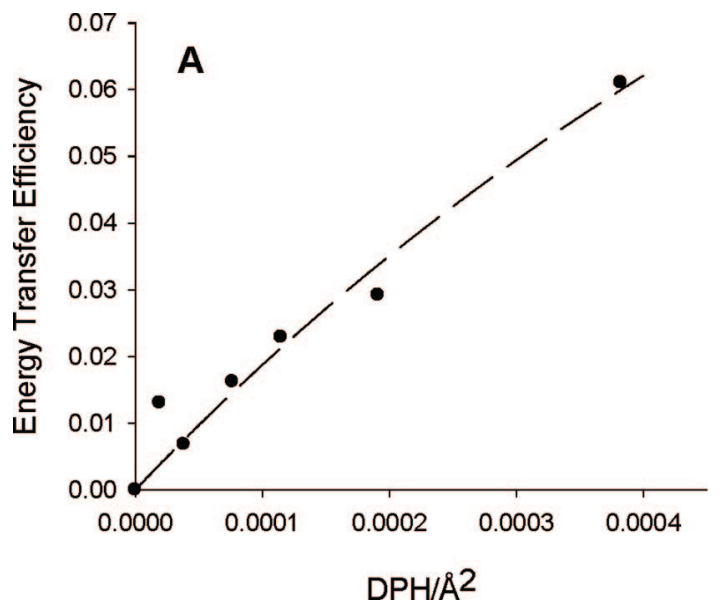

B

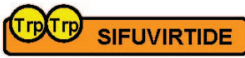

(TIT) SIFUVIRTIDE
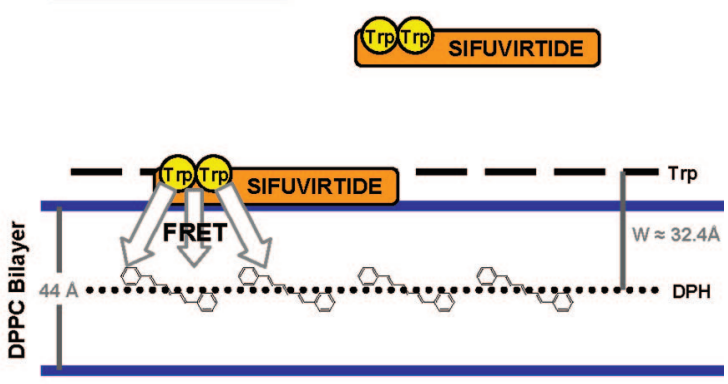

Figure 5. Energy transfer from Trp residues of sifuvirtide (donor) to DPH (acceptor) in DPPC vesicles (3 mM). (A) FRET efficiencies obtained at different proportions of DPH. (B) The schematic representation of the FRET assay suggests an adsorption of sifuvirtide on DPPC gel bilayers. FRET results suggest that the peptide is adsorbed at the surface of DPPC rigid membranes. $R_{0} \approx 30 \AA ; W=32.4 \AA ; x_{\mathrm{L}}$ (fraction in lipid) $\approx 0.25$. Peptide molecules located in the bulk aqueous phase (depicted at the top of the figure) are not quenched by FRET.

\section{Conclusions}

Sifuvirtide is a second generation HIV fusion inhibitor, which revealed promising results during the preclinical evaluation and clinical trials. ${ }^{8,9}$

In solution, sifuvirtide has a monomeric (or a small oligomeric) form, and no extensive oligomerization occurs until saturation. Therefore, this peptide can present clinical advantages in its administration, as well as in its molecular action to inhibit the virus entry.

The use of fluorescence spectroscopy-based methodologies revealed an unique selectivity of sifuvirtide to gel-phase membranes. At variance with other peptidic fusion inhibitors, such as enfuvirtide or T-1249, sifuvirtide does not partition into more fluid noncharged membranes. Enfuvirtide presents a high affinity for POPC fluid membranes, ${ }^{14}$ and T-1249, complementarily to its affinity to POPC fluid membranes, adsorbs at the surface of cholesterol-rich domains. ${ }^{15}$ Sifuvirtide is remarkably efficient in screening and adsorbing only to the rigid lipid platforms, as seen in the quenching and FRET experiments. On the other hand, it does not have a significant interaction neither with POPC fluid membranes nor cholesterol-rich membranes. This is compatible with its lower hydrophobicity, compared to both enfuvirtide and T-1249.

Although partitioning of enfuvirtide and T-1249 to membranes contributes to the high efficacy to inhibit the HIV entry into the cells, ${ }^{48}$ this phenomenon is not as selective as for sifuvirtide, considering the fact that the receptors essential to the fusion mechanism are localized in very rigid domains of the membrane. Lipid rafts (cholesterol and sphingomyelin-rich domains) are considered as essential for the virus entry. ${ }^{49}$ The rigidity of the domains is an important factor for the inhibition or the enhancement of the virus fusion capacity. Fluidification of the membrane by depletion of cholesterol and subsequent destruction of the rafts inhibit the HIV entry because of the loss of the signaling pathways and receptor functions..$^{50}$ On the other hand, turning the membrane more rigid, as shown in ceramide-rich membranes (ceramide platforms), also inhibits

(48) Castanho, M. A.; Fernandes, M. X. Eur. Biophys. J. 2006, 35, 92 103.

(49) Campbell, S. M.; Crowe, S. M.; Mak, J. J. Clin. Virol. 2001, 22, $217-$ 227.

(50) Nguyen, D. H.; Taub, D. D. Mol. Interv. 2004, 4, 318-320. the virus entry because of restrictions on the lateral diffusion of the receptors needed for the membrane fusion. ${ }^{51}$ It is known that the receptors on the cell used for the viral fusion (e.g., CD4) are associated to more-rigid neutral glycosphingolipid (GSL)rich microdomains. ${ }^{52,53}$ The existence of gel-phase microdomains inside rafts is quite controversial, in part because of the difficulties associated to their determination. Nevertheless, studies of sphingomyelin/cholesterol/ganglioside (or ceramide) ternary mixtures revealed the existence of gel-phase domains, rich in sphingomyelin and ganglioside (or ceramide), distinct and separated from the liquid-ordered sphingomyelin and cholesterol-rich domains. ${ }^{54-56}$ The presence of GSL-binding domains on the V3 domain of HIV envelope protein gp120 is an evidence of the importance of GSL microdomains for the infection of HIV into the immune cells. ${ }^{52,53}$ The affinity of the fusion inhibitor peptide $\mathrm{T}-1249$ to the liquid-ordered rafts explains its improved efficacy relative to enfuvirtide. The selectivity and targeting of sifuvirtide toward more-rigid gelphase domains, as seen in our results, is probably a key factor to explain the 20-fold increase of efficacy relative to enfuvirtide. Other factors include a higher half-life and a larger affinity to gp41, and a possible specific interaction with the viral membrane because of its high enrichment on DPPC (and other saturated phosphotidylcholines) relative to the host membrane. ${ }^{57}$

This study shows that there is a trend between fusion inhibitor efficacy and the ability of drug peptides to adsorb to rigid lipid domains. According to our results, we can hypothesize a molecular mode of action for sifuvirtide on the HIV target-cell membranes and/or on the viral membrane. Sifuvirtide's ability to screen the more-rigid domains of the membranes provides a

(51) Finnegan, C. M.; Rawat, S. S.; Cho, E. H.; Guiffre, D. L.; Lockett, S.; Merrill, A. H., Jr.; Blumenthal, R J. Virol. 2007, 81, 5294-5304.

(52) Hammache, D.; Yahi, N.; Maresca, M.; Pieroni, G.; Fantini, J. J. Virol. 1999, 73, 5244-5248.

(53) Puri, A.; Hug, P.; Jernigan, K.; Barchi, J.; Kim, H. Y.; Hamilton, J.; Wiels, J.; Murray, G. J.; Brady, R. O.; Blumenthal, R. Proc. Natl. Acad. Sci. U.S.A. 1998, 95, 14435-14440.

(54) Ferraretto, A.; Pitto, M.; Palestini, P.; Masserini, M. Biochemistry 1997, 36, 9232-9236.

(55) Silva, L. C.; de Almeida, R. F.; Castro, B. M.; Fedorov, A.; Prieto, M. Biophys. J. 2007, 92, 502-516.

(56) Castro, B. M.; de Almeida, R. F.; Silva, L. C.; Fedorov, A.; Prieto, M. Biophys. J. 2007, 93, 1639-1650.

(57) Brügger, B.; Glass, B.; Haberkant, P.; Leibrecht, I.; Wieland, F. T.; Kräusslich, H. G. Proc. Natl. Acas. Sci. U.S.A. 2006, 103, 2641-2646. 
local increased concentration of the drug at the fusion site. Consequently, this study may help explain the improved clinical efficacy of this peptide compared to that of other drugs from the same class.

Acknowledgment. The authors thank FusoGen (Tianjin, P. R. China) for a kind gift of sifuvirtide. The authors thank A. Salomé Veiga and Sónia T. Henriques for support and helpful discussions. Fundação para a Ciência e Tecnologia is acknowledged for funding (SFRH/BD/39039/2007 grant to H.G.F. and projects PTDC/QUI/69937/2006 and REEQ/140/BIO/2005).

Supporting Information Available: Appendix A: atomic-force microscopy of supported lipid bilayers. This material is available free of charge via the Internet at http://pubs.acs.org.

JA711247N 